\title{
Interaction-Driven Filling-Induced Metal-Insulator Transitions in 2D Moiré Lattices
}

\author{
Haining Pan and Sankar Das Sarma \\ Condensed Matter Theory Center and Joint Quantum Institute, \\ Department of Physics, University of Maryland, College Park, Maryland 20742, USA
}

\begin{abstract}
Using a realistic band structure for twisted $\mathrm{WSe}_{2}$ materials, we develop a theory for the interaction-driven correlated insulators to conducting metals transitions through the tuning of the filling factor around commensurate fractional fillings of the moiré unit cell in the 2D honeycomb lattice, focusing on the dominant half-filled Mott insulating state, which exists for both long- and short-range interactions. We find metallic states slightly away from half-filling, as have recently been observed experimentally. We discuss the stabilities and the magnetic properties of the resulting insulating and metallic phases, and comment on their experimental signatures. We also discuss the nature of the correlated insulator states at the rational fractional fillings.
\end{abstract}

The Fermi liquid (FL) theory is the most successful paradigm in condensed matter physics asserting that an interacting many-fermion system in dimensions higher than one (e.g., metals, normal He-3) has a bijection with the noninteracting Fermi gas. The low-energy quasiparticle excitations of the interacting Fermi system behave as almost noninteracting excitations of the Fermi gas with renormalized properties such as the effective mass. A well-known simple exception to the FL paradigm was pointed out by Wigner rather early [1] where he showed that strong long-range Coulomb interactions, would crystallize a continuum electron gas, creating a Wigner crystal (WC) of electrons, so that the Coulomb potential energy of the electrons is minimized instead of the kinetic energy as in the noninteracting or the weakly interacting system. Obviously, the WC is not a FL. Later, Mott [2] argued that interacting band electrons in a lattice would undergo correlation-driven metal to insulator transition for strong enough interactions. The Mott transition is adiabatically connected to the Wigner transition [3, 4]. The concept of Mott transition evolved over time eventually, becoming a sharply defined paradigm as the Mott-Hubbard ( $\mathrm{MH})$ metal-insulator transition (MIT) [5]. The modern view of the $\mathrm{MH}$ transition [68] involves the correlation-driven MIT at the half-filling of a narrow tight-binding band with the electrons being localized at lattice sites as local magnetic moments in an antiferromagnetic insulating (AFI) state. Such an AFI, existing precisely at the half-filling of the original noninteracting band, is called a Mott insulator (MI), and it arises from the strong short-range interactions present in the Hubbard model preventing the double occupancy of lattice sites, thus creating a purely on-site interaction driven insulating state. Such a MI is quite distinct from the WC in three specific ways: (1) it is independent of the electron density, and does not necessitate a lowdensity electron system as the WC requires; (2) it arises purely from short-range correlation effects in contrast to the WC arising from the long-range Coulomb interaction; (3) the MI happens precisely at the half-filling of the noninteracting band with the average inter-electron separation being equal to the effective lattice constant (i.e., one electron per unit cell). Although both the WC and $\mathrm{MI}$ are interaction-driven insulators, the MI is ubiquitous in strongly correlated narrow band systems [9] whereas the pure WC is rarely experimentally observed [10]. The current work studies the interplay between WC and MI phenomena driving (MIT) in the context of actual experiments in twisted 2D moiré systems based on van der Waals materials [11-21]. In particular, our focus is on the physics of the filling close to half, where a metallic state could exist at small doping away from the half-filled MI state. Such correlated metallic states themselves are interesting in addition to the considerable interest in the physics of MIT.

Recent experiments have identified correlated insulating states in twisted transition metal dichalcogenides (TTMD) based moiré 2D systems at filling factors $\nu=1$, $3 / 4,2 / 3,1 / 2,1 / 3,1 / 4$, etc. [11-17], where $\nu$ denotes the number of holes per moiré unit cell. In TTMD moiré systems, strong spin-orbit coupling produces doubly degenerate flat hole bands with narrow effective bandwidth leading to strong correlation effects as the kinetic energy is exponentially suppressed. In addition to the filling factor $\nu$, the system has two other tuning parameters affecting correlation effects: the twist angle $\theta$ determining the moiré unit cell size and the effective dielectric constant $\epsilon$ determining the Coulomb coupling as defined by $e^{2} /(\epsilon r)$ where $r$ is the interparticle distance. $\theta$ defines the moiré band structure, and $\epsilon$ defines the Coulomb coupling strength whereas $\nu$ defines the band filling.

We start with a MI at $\nu=1$, and investigate if correlated metallic states could exist in its neighborhood, as has recently been observed in transport measurements [11]. We note that the phase diagram of the TTMD at fixed rational fillings has recently been calculated [22] and experimentally studied $[15,17]$. The insulating states arising at various fractions (e.g., $\nu=1 / 3,1 / 2$, etc.) are neither strict MI nor strict WC. They are best described as 'correlated insulators' (CIs). They are Mott-like because they are commensurate with the moiré lattice and are thus connected to the band physics, but they are Wigner-like because their existence, except for the $\nu=1$ (MI), depends on long-ranged Coulomb potential. These 
are all CIs specific to moiré systems, which are neither MI nor WC.

The realistic interacting tight-binding Hamiltonian for TTMD-based moiré systems is [22, 23]

$$
\begin{aligned}
H & =\sum_{s} \sum_{i, j} t_{s}\left(\boldsymbol{R}_{i}-\boldsymbol{R}_{j}\right) c_{i, s}^{\dagger} c_{j, s} \\
& +\frac{1}{2} \sum_{s, s^{\prime}} \sum_{i, j} U\left(\boldsymbol{R}_{i}-\boldsymbol{R}_{j}\right) c_{i, s}^{\dagger} c_{j, s^{\prime}}^{\dagger} c_{j, s^{\prime}} c_{i, s},
\end{aligned}
$$

where the hopping terms $t_{s}$ represent band structures (depending on $\theta$ ), and effective interparticle Coulomb interactions $U$ represent the correlation effect (depending on $\epsilon$ ). Valley index $s$, spin-up or down, is coupled with $+K$ or $-K$ valley, respectively, in the Brillouin zone [23]. Both $t$ and $U$ involve distant nearest neighbors (i.e., our parametrization of Eq. (1) includes hopping up to the third nearest neighbors and Coulomb coupling term $U$ up to 1993 distant sites), refer to Refs. 22-25 for the motivation and derivation of Eq. (1) as the basic description for the interacting moiré physics in TTMD systems. Although the theory based on Eq. (1) should apply to all TTMD systems, our specific numerical results are for the $\mathrm{WSe}_{2}$ based TTMD structures currently being studied at Columbia University [11]. Details of the numerical model for Eq. (1) are available in Refs. 22 and 23. We emphasize that Eq. (1) cannot be thought of as either a Hubbard (or extended Hubbard) model or a WC (or generalized WC) model. Equation (1) is a semirealistic model for the actual interacting TTMD 2D moiré materials.

The 2D interacting problem in Eq. (1) is well defined, once all the hopping terms $t$ and interaction terms $U$, along with the filling factor $\nu$, are known. Obviously, the problem is insoluble exactly: the fermion sign problem and the 2D nature of the system make quantum Monte Carlo or exact diagonalization impossible. When the first term in Eq. (1) is zero, the problem has an exact classical solution which is obtained by minimizing the Coulomb energy (i.e., the second term). The exact classical solution for $t=0$ depends on the precise $\nu$ since the lattice symmetry of the classical state depends on $\nu$. Our strategy, as explained in Refs. 22 and 23 , is to use a selfconsistent mean-field (SCMF) theory starting with the classical WC solution as the initial input to obtain the final ground state of Eq. (1) in the presence of the hopping term. This is a reasonable strategy to search for CI ground states at rational fillings in the presence of strong interactions. It is possible that our theory overestimates the importance of non-FL correlated insulating states over FL conducting metallic states, which is acceptable since the problem is interesting only because of the breakdown of FL theory in the interacting system leading to the insulating states, which are absent (except trivially at band filling, $\nu=2$ ) in the tight-binding problem without interactions. For small deviations in $\nu$ around a rational filling, one can study the possible

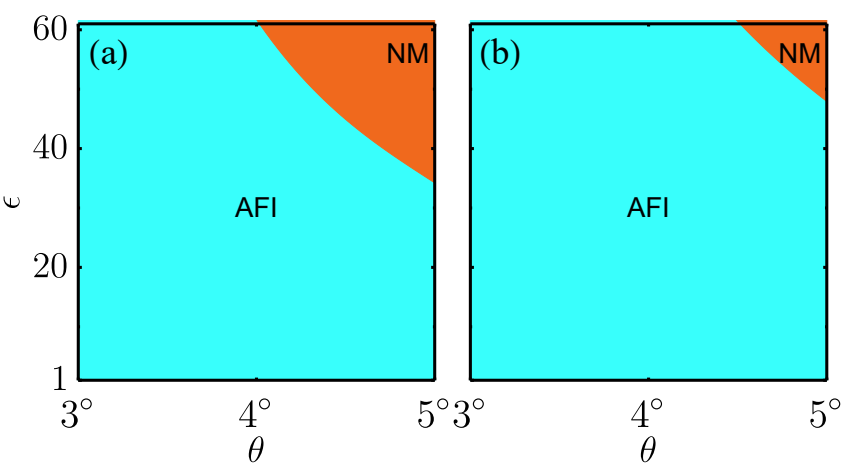

FIG. 1. The calculated phase diagram in the $\epsilon-\theta$ space at $\nu=1$ for (a) all distant neighbor Coulomb couplings, and (b) only the on-site $U$.

emergence of correlated metallic states by using a perturbation theory around the mean-field solution. Our current work is qualitatively different from earlier work since we are dealing here with metallic ground states in contrast to insulating ground states in [22, 23].

In Fig. 1, we show the calculated quantum phase diagram for the $\nu=1$ half-filled state in the $\theta-\epsilon$ space for the full long-range interaction with all distant neighbor Coulomb couplings as well as the effective Hubbard model keeping only the on-site $U$. The two phase diagrams are almost identical (except for some small quantitative differences), with an AFI state being the dominant phase except at large $\epsilon$ (i.e., weak interaction) and large $\theta$ (i.e., strong hopping) where the paramagnetic normal metallic (NM) FL shows up. This $\nu=1$ AFI is the wellknown MI phase. We emphasize that Fig. 1, manifesting a similar MI to NM transition for both long-range and on-site interaction models, serves as an important check on our theory. The fact that the theory reproduces the correct MI at $\nu=1$ irrespective of whether the interaction is long-ranged Coulomb or short-ranged Hubbard establishes the correct qualitative reliability of the theory.

Having established the model and its validity in predicting the correct MI at $\nu=1$, we now consider the main thrust of this Letter: filling-induced MIT in the flatband moiré system. We must distinguish among three distinct types of MIT predicted by the solutions to Eq. (1) in the moiré system. First, there are two types of correlationdriven MITs for fixed $\nu$, tuned respectively by $\epsilon$ and $\theta$, as apparent in Fig. 1. Increasing $\epsilon$ suppresses interaction and increasing $\theta$ increases the effective bandwidth, so tuning either $\epsilon$ or $\theta$ is an essentially equivalent way of changing the dimensionless interaction strength $U / t$, although, unlike in the simple Hubbard model, both $U$ and $t$ are represented by many effective parameters in Eq. (1) instead of a single parameter. Similar correlation-tuned $\epsilon-\theta$ phase diagrams are provided in Refs. 22 and 23 for $\nu=3 / 4,2 / 3,1 / 2,1 / 3,1 / 4$. We note that tuning the interaction strength in situ at a fixed $\nu$ is a challenge 


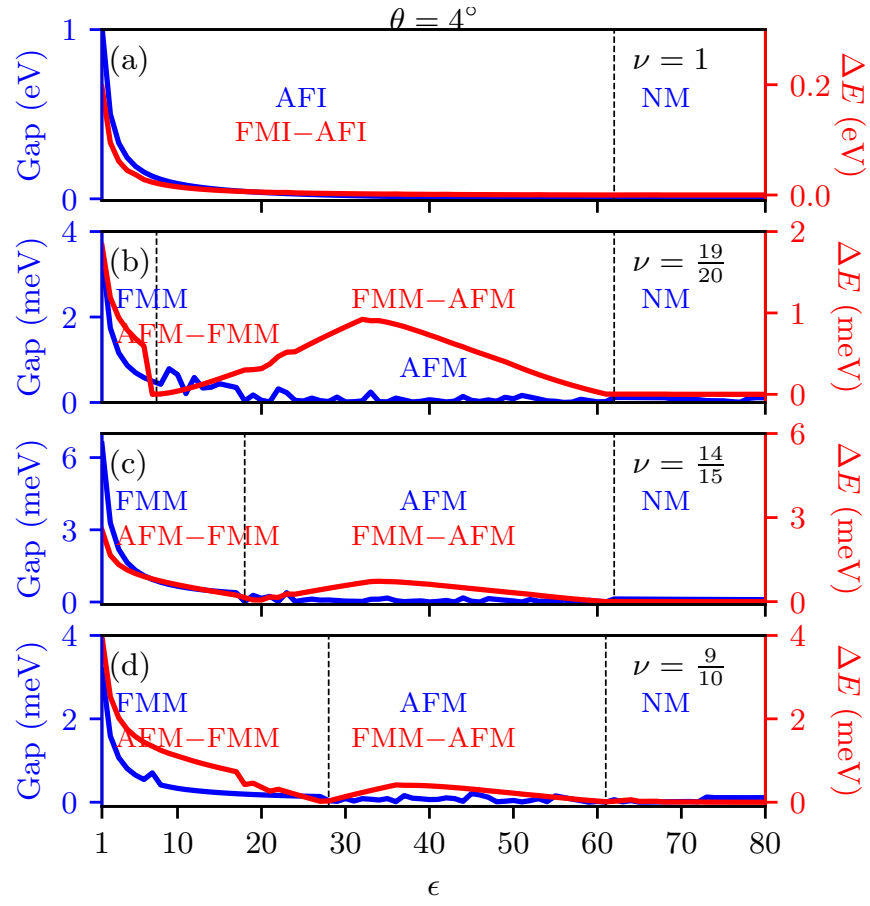

FIG. 2. The calculated charge gap (blue) as a function of $\epsilon$ for a fixed $\theta=4^{\circ}$ at (a) $\nu=1$; (b) $\nu=19 / 20$; (c) $\nu=14 / 15$; (d) $\nu=9 / 10$. The red line is the absolute energy difference between two competing phases. The first order transition happens at the vertical dashed line with the name of phases labeled in blue. AFI, AFM, FMI, FMM, and NM denote respectively the antiferromagnetic insulator, antiferromagnetic metal, ferromagnetic insulator, ferromagnetic metal, and normal paramagnetic metal.

experimentally since a typical sample has a fixed twist angle and substrate, and changing samples to change $\theta$ or $\epsilon$ may lead to other unknown modifications. Actually, $\epsilon / \theta$-tuned MIT has not yet been observed in TTMD [or twisted bilayer graphene (TBLG)], where a system is experimentally found to be either insulating or metallic depending on $\nu$ at low temperatures.

More interesting and accessible is the third type of MIT, which is tuned by $\nu$ at fixed $\epsilon$ or $\theta$. In the fillingtuned MIT, one dopes (or gates) the system away from a fixed $\nu$ where additional holes or electrons are created because of doping, and the system could undergo an insulator to metal transition solely because of doping (i.e., variation in $\nu$ ) itself without any explicit change in $\epsilon$ or $\theta$. Such $\nu$-tuned MIT has been reported in the $\mathrm{WSe}_{2}$ TTMD structures, where our theory should be applicable [11]. Our theory is partially motivated by the recent experiments at Columbia University [11], which is also recently observed in the twisted $\mathrm{WSe}_{2} / \mathrm{WSe}_{2}[26]$ and a similar heterostructure system $\mathrm{MoTe}_{2} / \mathrm{WSe}_{2}$ [27].

In Figs. 2(b)-2(d), we show calculated charge (energy) gaps at Fermi energy as a function of $\epsilon$ for fixed $\theta=4^{\circ}$ for several values of $\nu$, close to but slightly below $\nu=1$.
We also show the same quantity for $\nu=1$ for the sake of comparison in Fig. 2(a). Our calculation is perturbative starting from the $\nu=1 \mathrm{MI}$ which we calculate nonperturbatively using SCMF theory. The experimentally relevant value of $\epsilon$ depending on the sample and experimental details is approximately between 5 and 30 , most likely $10-15$. It is remarkable that although the $\nu=1$ state is a strong Mott AFI with a large gap for reasonable $\epsilon$, the situation is qualitatively different for $\nu$ just slightly below unity, where the ground state is a ferromagnetic metal (FMM) or antiferromagnetic metal (AFM) depending on $\epsilon$ in contrast to the antiferromagnetic MI at $\nu=1$. (The AFM phase is a spin density wave adiabatically connected to the Néel AFI at $\nu=1$.) This is the filling-induced MIT, where the states at $\nu=$ $1,2 / 3,1 / 2,1 / 3$, etc. are CI, but nearby states doped slightly away are metallic. The absolute energy difference between the two competing phases (AFM versus FMM) near $\nu \sim 1$ is in red corresponding to the right axis in Fig. 2. We mention that the small gap at small $\epsilon$ (i.e., strong interactions) in the effectively metallic ground state reflects that the calculation is always done at a rational filling factor, not at a true incommensurate filling which would be the generic experimental situation just slightly away from half-filling. This numerical gap in the metallic phase is fictitious due to the finite discretization of the momentum space, which should vanish as the mesh in the momentum space becomes finer, i.e., it is metallic in the thermodynamic limit. Note that this gap is orders of magnitude smaller than the calculated gap at $\nu=1$ and the gap goes quickly to zero with increasing $\epsilon$. This ensures that our theory captures the correct physics of generic metallicity at incommensurate filling close to $\nu \sim 1$, at small $\epsilon$ (large interaction), which approaches the limit where Nagaoka-like ferromagnetism dominates. For $\nu>1$, we find similar metallic state but without the Nagaoka ferromagnetism. This is because the filling denotes the doping of hole and it is equivalent to taking out the electrons if $\nu$ goes above 1 . In the electron number basis, the hopping is negative, where the Nagaoka theorem requires a positive hopping, thus the Nagaoka ferromagnetism is not guaranteed at $\nu>1$. Figure 3 shows the representative theoretical results. We note that the $\nu>1$ manifests an AFM phase in contrast to Nagaoka-like FMM for $\nu<1$.

The first transport experiment on $\mathrm{WSe}_{2}$ already showed the existence of a strong insulating phase at $\nu=1$ and metallic phases for doping slightly away from halffilling [11]. So, our finding of a filling-tuned MIT in TTMD moiré system around $\nu \sim 1$ is consistent with experimental results [26]. We have also checked that similar metallic phases exist around the other TTMD CIs at fractional occupancy e.g., $\nu=1 / 3$ [28].

Having established that a metallic state exists generically away from half-filling (and other rational) filling in the system, we now briefly discuss the nature of the 


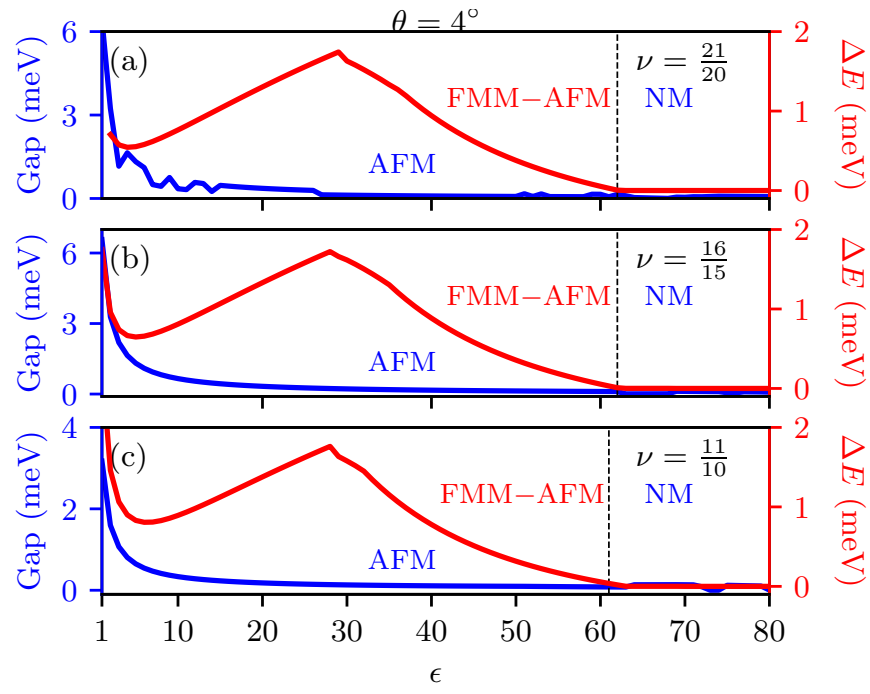

FIG. 3. The calculated charge gap (blue) as a function of $\epsilon$ for a fixed $\theta=4^{\circ}$ at (a) $\nu=21 / 20$; (b) $\nu=16 / 15$; (c) $\nu=11 / 10$. The red line is the absolute energy difference between closest competing ground states. Refer to Fig. 2 for notations.

correlated insulating states, which should all be metallic states in the noninteracting band picture because they represent fractionally filled single-particle energy bands of the TTMD system. We have already emphasized that the interaction driven insulating phase at fractional rational fillings $[22,23]$ arising in moiré TTMD materials should be thought of simply as a CI rather than as WCs or MH insulators, except at $\nu=1$ which is a strict MI. Quite generally, WC and MI are adiabatically connected - with the WC being the weak lattice potential and low-carrier-density (and consequently, the vanishing filling factor) limit of the CI whereas the MI is the strong lattice potential (and hence flatband) half-filling limit of the CI (in specific situations) [4]. For the half-filled $\nu=1$ situation, the MI description is the appropriate description because this insulator arises at half-filling independent of how high the carrier density might be. Additionally, as shown in Fig. 1, the $\nu=1 \mathrm{MI}$ exists independent of whether the interaction is long- or short-ranged. But the insulator at other fillings (e.g., $\nu=3 / 4,2 / 3,1 / 2,1 / 3$, $1 / 4$ as discussed in Ref. 22) cannot be a simple MI since they disappear for the on-site interaction-only model. We have explicitly checked that the insulating phase at all $\nu$ except for $\nu=1$ disappears if the distant neighbor interaction terms are zero in Eq. (1). The fact that the existence of CIs at $\nu$ other than 1 depends crucially on having a long-range interaction may indicate that the WC terminology is more appropriate for the CI for $\nu$ other than unity. But this is untrue as can be seen from Fig. 4 where we show the dimensionless continuum Coulomb coupling $r_{s}$, which is the average interparticle separation measured in units of the effective Bohr radius as a function of $\epsilon$ and $\nu$ for a fixed $\theta=4$ in Fig. 4(a) and as a function of $\theta$ and
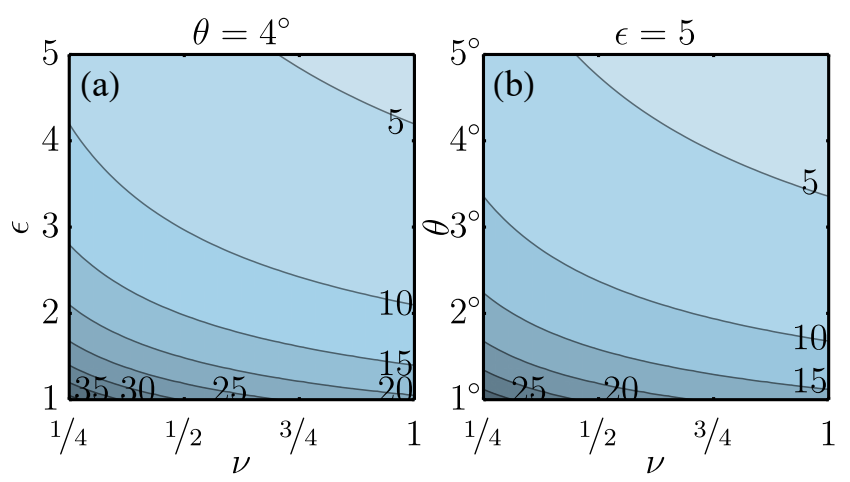

FIG. 4. The dimensionless continuum Coulomb coupling $r_{s}$ (a) at a fixed $\theta=4^{\circ}$; (b) at a fixed $\epsilon=5$. The lattice constant of the monolayer $\mathrm{WSe}_{2}$ is $3.28 \AA$ [30], and effective mass $m^{*}=0.45 m_{e}[31]$ ( $m_{e}$ is the rest electron mass). The $2 \mathrm{D}$ quantum WC condition $r_{s}>30$ is unsatisfied in the experimentally relevant regime.

$\nu$ for a fixed $\epsilon=5$ in Fig. 4(b). It is obvious that the applicable $r_{s}$ for TTMD are too small in the physical parameter regime (i.e., $\epsilon>5$ and $\nu>1 / 4$ ) for the $\mathrm{WC}$ to occur as it is well-established that the critical $r_{s}$ necessary for a $2 \mathrm{D} \mathrm{WC}$ is $r_{s}>30$ [29]. These CIs at simple rational fillings other than 1 are better considered as the lattice versions of a WC [i.e., quantum charge density wave ordering $(\mathrm{QCDW})]$. We predict that there should be a similar interaction-induced MI in TBLG at $\nu=1 / 8$, etc. (like $\nu=1 / 2$, etc. in TTMD), where electrons occupy distant TBLG moiré unit cells forming a QCDW.

Our finding that a TTMD half-filled doped hole system is an antiferromagnetic MI is significant since the corresponding TBLG CI ground states are deemed ferromagnetic Chern insulators [32-37]. Establishing the antiferromagnetic spin configuration of a TTMD half-filled moiré system is an important future experimental challenge.

We have theoretically discussed the filling-factor tuned MIT in 2D moiré TTMD hole doped materials as well as the nature of the correlated insulating states at rational fillings (which are all nominal metals in the noninteracting band pictures). We establish the existence of an antiferromagnetic Mott insulator at half-filling and the emergence of nearby (i.e., just away from half-filling) magnetic metallic phases. Our results are reminiscent of the Nagaoka ferromagnetism inherent in the strongly interacting Hubbard model around half-filling although we consider finite (albeit low) doping level [38], and are consistent with recent experiments reporting metallicity in TTMD slightly away from half-filling $[26,27]$. We predict such metal-insulator transitions at other rational fillings such as $\nu=1 / 3$ [28].

We gratefully thank Cory Dean and Abhay Pasupathy (and their team members, especially Augusto Ghiotto) for discussions on their unpublished experimental results, 
and Fengcheng $\mathrm{Wu}$ for helpful discussion. This work is supported by the Laboratory for Physical Sciences.

[1] E. Wigner, Phys. Rev. 46, 1002 (1934).

[2] N. F. Mott, Proc. Phys. Soc. A 62, 416 (1949).

[3] W. Kohn, Phys. Rev. Lett. 19, 789 (1967).

[4] D. Vu and S. Das Sarma, Phys. Rev. Research 2, 023060 (2020).

[5] J. Hubbard, Proceedings of the Royal Society of London. Series A. Mathematical and Physical Sciences 276, 238 (1963).

[6] N. F. Mott, Rev. Mod. Phys. 40, 677 (1968).

[7] F. Gebhard, The Mott Metal-Insulator Transition: Models and Methods, Springer Tracts in Modern Physics (Springer-Verlag, Berlin Heidelberg, 1997).

[8] N. Mott, Metal-Insulator Transitions (CRC Press, 2004).

[9] M. Imada, A. Fujimori, and Y. Tokura, Rev. Mod. Phys. 70, 1039 (1998).

[10] C. C. Grimes and G. Adams, Phys. Rev. Lett. 42, 795 (1979).

[11] L. Wang, E.-M. Shih, A. Ghiotto, L. Xian, D. A. Rhodes, C. Tan, M. Claassen, D. M. Kennes, Y. Bai, B. Kim, K. Watanabe, T. Taniguchi, X. Zhu, J. Hone, A. Rubio, A. N. Pasupathy, and C. R. Dean, Nature Materials 19, 861 (2020).

[12] E. C. Regan, D. Wang, C. Jin, M. I. Bakti Utama, B. Gao, X. Wei, S. Zhao, W. Zhao, Z. Zhang, K. Yumigeta, M. Blei, J. D. Carlström, K. Watanabe, T. Taniguchi, S. Tongay, M. Crommie, A. Zettl, and F. Wang, Nature 579, 359 (2020).

[13] Z. Zhang, Y. Wang, K. Watanabe, T. Taniguchi, K. Ueno, E. Tutuc, and B. J. LeRoy, Nature Physics 16, 1093 (2020).

[14] Y. Tang, L. Li, T. Li, Y. Xu, S. Liu, K. Barmak, K. Watanabe, T. Taniguchi, A. H. MacDonald, J. Shan, and K. F. Mak, Nature 579, 353 (2020).

[15] Y. Xu, S. Liu, D. A. Rhodes, K. Watanabe, T. Taniguchi, J. Hone, V. Elser, K. F. Mak, and J. Shan, Nature 587, $214(2020)$.

[16] X. Huang, T. Wang, S. Miao, C. Wang, Z. Li, Z. Lian, T. Taniguchi, K. Watanabe, S. Okamoto, D. Xiao, S.-F. Shi, and Y.-T. Cui, Nature Physics 17, 715 (2021).

[17] C. Jin, Z. Tao, T. Li, Y. Xu, Y. Tang, J. Zhu, S. Liu, K. Watanabe, T. Taniguchi, J. C. Hone, L. Fu, J. Shan, and K. F. Mak, Nature Materials 20, 940 (2021).

[18] Y. Cao, V. Fatemi, S. Fang, K. Watanabe, T. Taniguchi, E. Kaxiras, and P. Jarillo-Herrero, Nature 556, 43
(2018)

[19] Y. Cao, V. Fatemi, A. Demir, S. Fang, S. L. Tomarken, J. Y. Luo, J. D. Sanchez-Yamagishi, K. Watanabe, T. Taniguchi, E. Kaxiras, R. C. Ashoori, and P. JarilloHerrero, Nature 556, 80 (2018).

[20] M. Yankowitz, S. Chen, H. Polshyn, Y. Zhang, K. Watanabe, T. Taniguchi, D. Graf, A. F. Young, and C. R. Dean, Science 363, 1059 (2019).

[21] X. Lu, P. Stepanov, W. Yang, M. Xie, M. A. Aamir, I. Das, C. Urgell, K. Watanabe, T. Taniguchi, G. Zhang, A. Bachtold, A. H. MacDonald, and D. K. Efetov, Nature 574, 653 (2019).

[22] H. Pan, F. Wu, and S. Das Sarma, Phys. Rev. B 102, 201104 (2020).

[23] H. Pan, F. Wu, and S. Das Sarma, Phys. Rev. Research 2, 033087 (2020).

[24] F. Wu, T. Lovorn, E. Tutuc, and A. H. MacDonald, Phys. Rev. Lett. 121, 026402 (2018).

[25] F. Wu, T. Lovorn, E. Tutuc, I. Martin, and A. H. MacDonald, Phys. Rev. Lett. 122, 086402 (2019).

[26] A. Ghiotto, E.-M. Shih, G. S. S. G. Pereira, D. A. Rhodes, B. Kim, J. Zang, A. J. Millis, K. Watanabe, T. Taniguchi, J. C. Hone, L. Wang, C. R. Dean, and A. N. Pasupathy, arXiv:2103.09796 (2021).

[27] T. Li, S. Jiang, L. Li, Y. Zhang, K. Kang, J. Zhu, K. Watanabe, T. Taniguchi, D. Chowdhury, L. Fu, J. Shan, and K. F. Mak, arXiv:2103.09779 (2021).

[28] "See supplemental Material for the results of $n u=1 / 3$.".

[29] N. D. Drummond and R. J. Needs, Phys. Rev. Lett. 102, 126402 (2009).

[30] A. Kormányos, G. Burkard, M. Gmitra, J. Fabian, V. Zólyomi, N. D. Drummond, and V. Fal'ko, 2D Mater. 2, 022001 (2015).

[31] B. Fallahazad, H. C. P. Movva, K. Kim, S. Larentis, T. Taniguchi, K. Watanabe, S. K. Banerjee, and E. Tutuc, Phys. Rev. Lett. 116, 086601 (2016).

[32] A. L. Sharpe, E. J. Fox, A. W. Barnard, J. Finney, K. Watanabe, T. Taniguchi, M. A. Kastner, and D. Goldhaber-Gordon, Science 365, 605 (2019).

[33] M. Serlin, C. L. Tschirhart, H. Polshyn, Y. Zhang, J. Zhu, K. Watanabe, T. Taniguchi, L. Balents, and A. F. Young, Science 367, 900 (2020).

[34] F. Wu and S. Das Sarma, Phys. Rev. Lett. 124, 046403 (2020).

[35] F. Wu and S. Das Sarma, Phys. Rev. B 102, 165118 (2020).

[36] N. Bultinck, S. Chatterjee, and M. P. Zaletel, Phys. Rev. Lett. 124, 166601 (2020).

[37] Y. Alavirad and J. Sau, Phys. Rev. B 102, 235123 (2020).

[38] Y. Nagaoka, Phys. Rev. 147, 392 (1966). 